\title{
PsyExper: Another experimental generation system for the IBM PC
}

\author{
KEVIN J. HAWLEY \\ University of Utah, Salt Lake City, Utah \\ (Student Award Winner for 1990)
}

\begin{abstract}
A system for creating and running psychological experiments on IBM PC compatible computers is described. The system attempts to reconcile the demand for flexible and powerful experimental software with the need for systems that are easy to understand and modify. Experiments are created by building a series of displays with variable compositions, durations, and stimulus onset asynchronies. The number, type, location, color, and perceptibility of stimuli within these displays can be systematically varied. Reaction time and other response measures can be collected from the keyboard, a voice-activated switch, or other external switches. The system is assembled from a series of Turbo Pascal and Turbo $\mathrm{C}$ routines that may be modified by the user or incorporated into new routines. Custom modules can be added easily to any of the system's menus. The system is best suited for discrete trial experiments with target detection, perceptual priming, lexical decision, or recognition memory tasks.
\end{abstract}

Several software systems designed to eliminate the need for programming in creating and running psychology experiments are now available. The power and sophistication of these systems varies widely. While some systems specialize in a few specific paradigms (e.g., CEDATS; see Eamon, 1982), others allow a wide variety of paradigms to be assembled from a set of allowable manipulations (e.g., MEL; see Schneider, 1988). Unfortunately, the ease of use of such systems tends to vary inversely with their power and sophistication (see Butler, 1988, for a review). Furthermore, even the most flexible system cannot anticipate the entire range of experiments that researchers may seek to perform. The drawbacks of these systems highlight two crucial problems for software developers who create experimental generation systems. First, any system that allows the development and modification of experiments without the need for programming must balance the demand for flexibility with the need for systems that are easy to use and understand. Second, since it is impossible to anticipate all the tasks an experimenter

\footnotetext{
Portions of this work were supported by a University of Utah Graduate Research Fellowship to the author and by Air Force Office of Scientific Research Grant 89-0275 to William Johnston at the University of Utah. I would like to thank Thayne Cooper of the Unisys Corporation for help in developing the interface card and some of the timer algorithms, Bill Johnston for advice and encouragement, and the many undergraduate assistants at the University of Utah who helped in ferreting out the "bugs" in earlier versions. I also wish to acknowledge Veronica Dark, whose postdoctoral work provided much initial inspiration. Requests for reprints should be addressed to Kevin Hawley, Department of Psychology, University of Utah, Sait Lake City, UT 84112.

Note: The author or institution has a direct financial interest in the hardware or software described in this paper.-Editor
}

may seek to perform, these systems need to be designed so that they will be easy to expand or modify.

The system described in this paper, PsyExper, represents an attempt to strike a balance between these conflicting demands. PsyExper is a collection of programming routines for IBM PC machines (or fully compatible clones) that allows psychologists to create and edit experiments, collect data, and prepare data for analysis without any programming. These routines are organized within a software "shell" that utilizes many of the userfriendly features found in expensive professional software packages. These include a powerful text editor, "help" files, menu-driven program selection, and a DOS interface that allows a user to perform any legal DOS command (such as printing or copying files) and to execute external programs (such as statistics or graphics packages) without leaving the system. More importantly, PsyExper employs an open programming architecture, making it easy to add custom modules to the system. These modules can incorporate any of the routines contained in the system library (or any custom routines supplied by the experimenter) and can be added to any of the system's menus with just four lines of code. The system is best suited for developing discrete trial paradigms for target detection (see Treisman \& Gelade, 1980), perceptual priming (see Feustel, Shiffrin, \& Salasoo, 1983), lexical decision (see Humphreys, Evett, \& Taylor, 1982), and memory dissociation (see Jacoby \& Dallas, 1981) tasks.

\section{THE PSYEXPER SYSTEM}

PsyExper is divided into two functionally separate units-namely, the data collection programs and the supporting utility software. All of the data collection routines 
are written in Turbo $\mathrm{C}$ (Version 1.5), with certain timecritical routines for XT-class machines written in 8088 assembly language. The utility software is written in Turbo Pascal (Version 4.0); with the exception of the built-in editor and the DOS system interface, it consists of routines to aid the assembly of experimental files and analysis of the collected data.

The basic unit of presentation in PsyExper is the display (see Table 1). In general, a display may be composed of up to 20 stimuli. A stimulus is defined as a string of $1-10$ characters, and it may occur at any one of 20 userdefined screen locations. Stimuli longer than 10 characters (e.g., long words or sentences) may be created by making some of these screen locations adjacent and then writing the appropriate characters to each location. The number, type, color, and location of stimuli in any given display may be varied, as well as the duration and stimulus onset asynchrony (SOA) of the display as a whole. Furthermore, all stimuli in a display may be backward masked with a pattern specified by the experimenter, and the display itself can appear against any 1 of 10 userdefined backgrounds. All stimuli, masks, and backgrounds are built from characters in the standard ASCII extended character set. Under certain circumstances, these characters can be modified so that users can create characters of their own choosing. This allows for the presentation of limited forms of black and white graphics with a $640 \times 200$ pixel resolution. If the default BIOS characters are used, the stimuli, masks, and background displays may each be presented in any of 16 colors, while the background screen may be any of 8 colors. Finally, the perceptual clarity of stimuli can be varied by presenting them in conjunction with a random pattern of dots, which can be systematically removed at a rate specified by the experimenter.

Table 1

A Layout of the Information Contained in a Display in PsyExper (As It Is Actually Represented in a Gen file)

\begin{tabular}{crrrrrr}
\hline 1 & 2 & 6 & 12 & 1 & 0 & 65 \\
1 & 1 & 3 & 0 & & & \\
HOUSE & & & & & \\
2 & 1 & 4 & 0 & & & \\
TREE & & & & & \\
\hline
\end{tabular}

Note-The first line of each display contains information that is global to the display as a whole. From left to right, this information is the trial number (i.e., the ordinal position of the display within the experiment), the number of individual stimuli contained in the display, the exposure duration and SOA (in refresh cycles), the background display (1-10) against which this display should appear, a flag denoting whether the display should be preceded by a warning signal, and the ASCII code of the correct keyboard response for this display (not all of this information is necessarily used in any one experiment). The next two pairs of lines contain the particular stimuli to be displayed (i.e., HOUSE and TREE) preceded by information regarding the individual characteristics of these stimuli. Again, from left to right, this information is the particular screen location (1-20) at which each stimulus should appear, the color $(0-15)$ of that stimulus, its type (a generalized flag for identifying experimental classes of stimuli), and a flag denoting whether a warning signal should precede the onset of this stimulus in this particular location.
All displays in the PsyExper system are stored in a stimulus generation, or Gen, file. The order of displays within a Gen file determines their order of presentation. Displays are read into the computer's memory in groups of 100 , so that up to 100 displays can be presented in succession without access to the disk. Although the total number of displays that may be presented during an experiment is limited only by the amount of disk space, the package currently limits the number of displays for which data are collected to 2,000. Multiple Gen files may be used in an experiment, and each of these may optionally be preceded by a computer-controlled display of text contained in a file specified by the experimenter. Although this feature was originally intended to facilitate the presentation of instructions for each phase of an experiment, it may also be used to present text for studies of reading comprehension.

\section{Conducting an Experiment}

Experimental assembly. Preparing an experiment in PsyExper requires the construction of at least two input files. The first is the Gen file (or files), which contains the displays to be presented during the experiment. Gen files are assembled by building a skeleton file, which contains the individual displays of the experiment without the actual stimuli, and then systematically mapping lists of stimuli into particular locations of any display in that skeleton file. Since all stimuli in a display are individually tagged, they may be systematically rotated through the various conditions/displays of an experiment. This arrangement also allows the experimenter to manipulate the individual characteristics (e.g., color, location, etc.) of the stimuli in a display. A skeleton Gen file may be assembled in one of two ways. First, the experimenter may build the individual displays by hand, entering values for the duration, SOA, and stimulus characteristics for each display in response to prompts from the system. Although this method provides a high level of flexibility in controlling the assembly of an experiment, it can entail a lot of typing. The second method of assembling a skeleton file involves the use of an "intelligent" file builder contained in the system. This routine prompts the experimenter for the different display characteristics to be manipulated and then constructs a complete set of counterbalanced displays from all possible combinations of those characteristics. ${ }^{1}$ All of the displays created by either method may be assigned either randomly or in blocks to different orders of presentation during an experiment. Additional utilities are provided to aid in randomizing the stimuli within a display, merging Gen files, or changing any of the characteristics of a display.

The second file that is required for data collection is an Experimental values, or Exvals, file. This file contains a listing of the global parameters in effect for a particular experiment. Exvals files are assembled by responding to a series of system prompts in one of the utility programs. For example, the system will ask the experimenter 
to identify the screen coordinates for each of the 20 possible locations at which stimuli may appear, and to describe each of the 10 possible background displays that may be shown during an experiment. For studies in which the keyboard will be used to collect subject responses, a listing of legal keypress responses may be elicited. More importantly, Exvals files contain a listing of the Gen files to be used in a study and the name of the output file in which a subject's data is to be stored. A complete listing of the information contained in an Exvals file is given in the "help" files contained in the system.

Collecting data. Data collection is accomplished by selecting one of the three programs provided with the system from the data collection menu and entering the name of an Exvals file and a three-digit subject number at a system prompt. The subject number is appended to the output file name provided in the Exvals file to generate a unique output file for each subject. Although the data collection programs are very similar in overall construction and function, each one provides a somewhat different set of potential manipulations and measurements. The first program allows for individual stimuli to be displayed behind a masking pattern of random dots. This pattern mask may contain from 1 to 500 dots, which can be removed by the computer (thus making the stimulus more visible) at the average rate of 1 dot every $1-32,767 \mathrm{msec}$. Both the number of dots in the mask and the rate at which they are removed are specified by the experimenter in the Exvals file. Reading latencies (in milliseconds) can be collected for these stimuli via a voice-activated switch interfaced with the system. The second data collection program allows the experimenter to identify a "correct" keypress response for any particular display and then measures the latency and accuracy of a subject's response for that display. The latency measurements for this program are collected by means of Emerson's (1988) timex function, with the resolution of this measurement (i.e., milliseconds, centiseconds, etc.) set by the experimenter. ${ }^{2}$ Both of these first two data collection programs feature a usermodifiable character set, and they are thus capable of displaying limited black and white graphics. The third data collection program is a duplicate of the second, except that it allows for stimuli to be displayed in different colors. This ability to manipulate color is purchased at the cost of the ability to modify the character set.

Display exposure durations in each of the three data collection programs are defined in refresh cycles $(16.67 \mathrm{msec}$ in countries with $60-\mathrm{Hz}$ power; $20 \mathrm{msec}$ in countries with $50-\mathrm{Hz}$ power). Displays are moved to the screen by writing their contents directly to video memory. Although this process is timed so that each display appears at the start of a video refresh cycle, the actual onset time for any particular stimulus will vary as a function of its screen position. The lag for a particular stimulus' onset time can be estimated as $0.67 \mathrm{msec}$ for each line between the stimulus' position and the top of the screen. The minimum time between successive displays is approximately $16 \mathrm{msec}$. This means that the minimum possible exposure duration for backward-masked displays is $33 \mathrm{msec}$ (16.67 msec illumination plus a $16.67-\mathrm{msec}$ interval before the onset of the mask display). The actual time may vary as a function of the experimental options selected by the experimenter. For example, if a complex background display is to be changed prior to the onset of a particular display, the actual interdisplay interval may be much longer.

Finally, all three data collection programs can present up to two queries (the contents of which are specified by the experimenter in the Exvals file) following a test display. Subjects can respond to these queries either from the keyboard or from a set of external switches, which can be interfaced with the system. Furthermore, if a monochrome (TTL) monitor is added to the system, the data collection programs can optionally display information regarding the contents of the subject's screen on this monitor. This feature allows the experimenter to monitor a subject's performance, and facilitates the scoring of reading accuracy.

Data reduction. The PsyExper system includes a number of data reduction routines designed to perform simple data summaries and help prepare data for analysis via some other statistical package. Data reduction consists of at least two steps. During the first step, individual data points in a subject's data file are identified with a set of condition codes that denote the particular combination of within-subjects factors in effect when that datum was collected. This is accomplished by merging the subject data file with a file containing the corresponding condition codes. The condition code file can be built either by hand or with the aid of a routine that scans a Gen file for particular display parameters identified by the experimenter. The system is also capable of identifying certain subjectdefined variables, such as report accuracy for an item during the study phase of a study-test experiment. This allows the experimenter to segregate items in the test phase into items that were or were not correctly reported during the study phase.

The second step of the data reduction process consists of summarizing data with similar condition codes either across subjects or across stimuli (i.e., subject- or stimulusbased analyses). The routines that perform these summaries produce mean latencies of response, mean accuracies, and tallies of the number correct, and total number of data points observed. For the subject-based routines, this process produces a set of summaries for all combinations of conditions observed for that subject. This table can then be analyzed with any statistical package that supports either free-field or fixed-format data input (e.g., SPSS or BMDP). For the stimulus-based routines, only a single set of summary statistics, for a single combination of conditions, is produced. However, multiple passes with this procedure can yield summaries across several combinations of conditions. These summaries can be com- 
bined to produce a table similar to that described for the subject-based routines. In general, both the subject- and the stimulus-based routines will accommodate up to four between-subjects variables with up to 99 levels each, and up to five within-subjects factors with 9 levels each.

\section{Hardware Requirements}

The PsyExper system requires an IBM PC (XT or AT) or a truly compatible clone with at least $384 \mathrm{~K}$ RAM, a hard disk, and a color graphics adaptor (CGA) card. More sophisticated graphics cards (e.g., EGA, VGA, etc.) may be used, as long as they provide CGA emulation. Portions of the package will not run on certain clones (e.g., the Sperry PC) that lack the standard internal clock. In addition, some of the data collection programs require the installation of a custom interface/timer card in order to be able to access data from voice keys and external switches. This card is similar (but not identical) to that described by Lavond and Steinmetz (1989); a schematic is provided in the manual accompanying the system. The only additional software required by the system is DOS 3.0 or higher, and, optionally, a statistical or graphics package. Optional equipment includes a monochrome monitor with a Hercules compatible interface card (TTL), a voice-activated switch (see Hawley \& Izatt, 1991) and/or external switches, headphones, and a printer.

\section{Additional Features}

The PsyExper package includes a manual with user and reference guide sections that provide details concerning the organization and layout of the package, use of the menu system, and a command reference for the built-in editor. In addition, a listing of terms used in the package, a table of error messages, and detailed instructions regarding modification of the internal character set are provided. Three sample experiments are included to help users become better acquainted with some of the package's capabilities. Schematics for all custom hardware that may be required by the system are also included.

For users who are interested in modifying the system, separate purchase of the source code includes a set of Turbo Pascal units containing all of the basic procedures and functions used in assembling the utility software (an equivalent set of files containing the Turbo $\mathrm{C}$ code for the data collection programs is also provided). These routines may be used to modify existing portions of the system or to assemble entirely new routines. A programmer's guide describing the use and construction of each of these routines is contained in the manual.

\section{System Limitations}

PsyExper's most serious limitation is its exclusive focus on discrete trial paradigms. In its current form, the system cannot conduct studies in which experimental trials must be modified on the basis of a subject's performance (e.g., operant conditioning experiments). Although the open architecture of the system would permit the addition of routines to facilitate such tasks, the necessary modifications could be quite extensive. Since packages to implement these paradigms already exist (e.g., MEDPC; see Tatham \& Zurn, 1989), many users may find this modification too extensive to pursue.

There are also a number of structural limitations in the system. The most serious of these is the limit of 2,000 data that may be collected, as well as the limit of 100 displays that may be presented without access to the disk. These problems are related, in that they reflect an underutilization of dynamic memory allocation techniques. An enhancement of the system is planned to reduce these problems. Second, the system's exclusive reliance on CGA mode for stimulus presentation limits the overall resolution of stimuli, as well as its ability to manipulate graphics. The original PsyExper system employed a CGA display protocol both because that was the default standard in our lab and because it was the lowest common denominator in terms of screen adaptors that would ensure the correct functioning of the system across the widest range of machines. An update of the system is planned to take advantage of the capabilities of more advanced graphics display cards. This will include the ability to draw displays with a graphics package such as PC PAINT and then incorporate the displays into an experiment.

\section{SYSTEM COMPARISONS}

PsyExper has a variety of strengths and weaknesses in comparison with other experimental generation systems that are currently available. The following comparisons were based on information contained in the published summaries of the systems in question and on a review of these systems by Butler (1988). The interested reader should refer to the manuals for all systems mentioned in this section for more information regarding their capabilities and limitations.

There are two basic systems for IBM PC machines with capabilities similar to PsyExper's. These are APT PC (Poltrock \& Foltz, 1988) and MEL (Schneider, 1988). All three experimental systems allow stimuli to be constructed from characters in the standard ASCII character set. Both PsyExper and MEL allow the use of computer graphics, but MEL additionally allows the use of computer-generated tones and animation. Like MEL, the PsyExper system allows parts of displays and the durations of individual displays to be defined as variables. Moreover, stimuli in both systems can be "tagged" and systematically substituted for one another. This allows for the fast development of a variety of experimental designs. Although both MEL and PsyExper can be modified with user-written routines, the overall construction of PsyExper (using subroutines written in Pascal or $\mathrm{C}$ as opposed to a fourth-generation language that automatically generates object code for the experimenter) is probably more conducive to user modification than MEL is.

With respect to data collection, all three systems allow for the collection of response latencies and accuracies from keypress data entered by subjects. In addition to this, both 
MEL and PsyExper allow for data collected from external devices such as voice keys and external switches. PsyExper is probably more convenient in this regard, in that, unlike MEL, it does not require additional programming to make use of these devices. However, whereas both MEL and APT PC allow for the entry of string data in response to a stimulus, PsyExper does not. For data analysis, MEL is clearly the leader. Although all three systems provide both subject- and stimulus-based analyses, MEL provides a wide range of additional data analysis and graphics routines. PsyExper is perhaps slightly more versatile than APT PC, in that it allows data used in its analyses to be "filtered" by excluding data or subjects identified as unreasonable by the experimenter.

\section{CONCLUSION}

PsyExper is an experimental generation system for IBM PC type machines that allows the development of psychological experiments without programming. This system was designed to reconcile the demand for flexible and powerful software with the need for systems that are easy to understand and modify. With respect to experimental power, the system probably falls in between APT PC and MEL in terms of its overall experimental flexibility and ease of use. Although the system lacks some features provided by these other systems (e.g., string data input), it is comparable in others (e.g., ease of experimental assembly) and extends their capabilities in certain critical respects (e.g., use of a dot clearing paradigm). With respect to its overall construction, the present system's use of two readily accessible programming languages (Turbo $\mathrm{C}$ and Turbo Pascal), along with its use of a systematized collection of software routines, allows modifiability not readily found in other systems. These features make the PsyExper system a serious alternative to other experimental systems.

\section{AVAILABILITY}

Rights to the PsyExper system are held by the University of Utah. The system may be purchased for $\$ 100$ by contacting Arundeep Pradhan at the Technology Transfer Office of the University of Utah, 295 Chipeta Way, Suite 280, Salt Lake City, UT 84108 . The source code is available for an additional $\$ 75$. When ordering, please specify whether you are using an XT or an AT (or higher) system, and whether the system should be sent on 5.25 or 3.5-in. disks.

\section{REFERENCES}

BUTLER, D. L. (1988). A critical evaluation of software for experiment development in research and teaching. Behavior Research Methods, Instruments, \& Computers, 20, 218-220.

EAMON, D. B. (1982). CEDATS: A cognitive experimental design and testing system. Behavior Research Methods, Instruments, \& Computers, 14, 142-145.

EMERSon, P. L. (1988). TIMEX: A simple IBM AT C language timer with extended resolution. Behavior Research Methods, Instruments, \& Computers, 20, 566-572.

Feustel, T. C., Shiffrin, R. M., \& Salasoo, A. (1983). Episodic and lexical contributions to the repetition effect in word identification. Joumal of Experimental Psychology: General, 112, 309-346.

HAWLEY, K. J., \& IZATT, G. M. (1991). An inexpensive voice-activated switch for experiments involving vocal response times. Manuscript in preparation.

Humphreys, G. W., Evett, L. J., \& TAylor, D. E. (1982). Automatic phonological priming in visual word recognition. Memory \& Cognition, 10, 576-590.

JACOBY, L. L., DALLAS, M. (1981). On the relationship between autobiographical memory and perceptual learning. Joumal of Experimental Psychology: General, 3, 306-340.

LAvond, D. G., Steinmetz, J. E. (1989). An inexpensive interface for the IBM PC/XT and compatibles. Behavior Research Methods, Instruments, \& Computers, 21, 435-440.

Poltrock, S. E., \& Foltz, G. S. (1988). APT PC and APT U: Experiment development systems for the IBM PC and Apple II. Behavior Research Methods, Instruments, \& Computers, 20, 201-205.

SCHNEIDER, W. (1988). Micro Experimental Laboratory: An integrated system for IBM PC compatibles. Behavior Research Methods, Instruments, \& Computers, 20, 206-217.

TAtham, T. A., \& ZURN, K. R. (1989). The MED-PC experimental apparatus programming system. Behavior Research Methods, Instruments, \& Computers, 21, 294-302.

Treisman, A., Gelade, G. A. (1980). A feature integration theory of attention. Cognitive Psychology, 14, 107-141.

\section{NOTES}

1. There are certain restrictions on the type and overall number of display combinations that can be generated at one time with the autocreate feature. These limitations can usually be overcome through the creation of several subfiles with displays having the desired characteristics; these subfiles are then merged, using one of the file utilities provided in the package.

2. There are certain limitations on the accuracy of latencies collected with the timex function on IBM XT compatible machines. Specifically, the function's execution time can vary unpredictably between 0.2 and $28 \mathrm{msec}$, thus inflating latency measurements by an average of $14.2 \mathrm{msec}$. See Emerson (1988) for a complete description of this function and its limitations. 